\title{
The Application of Artificial Intelligence Technology in the Control of Electrical Automation Technology
}

\author{
Meifang Li \\ Heilongjiang College of Construction
}

\begin{abstract}
Keywords: Artificial intelligence technology; Electrical automation
\end{abstract}
\begin{abstract}
The application of artificial intelligence technology in the control of electrical automation technology is more and more widely. Intelligent control and intelligent fault processing can greatly improve the production quality and efficiency, and reduce the production cost and risk. In this paper, the author expounds the definition and application characteristics of artificial intelligence technology, analyzes the advantages and practical application of the combination with the control of electrical automation technology.

Electrical automation is a new product in the field of electrical information, and the main research contents of this subject include: system operation related to electrical engineering, information processing, power electronic technology, experiment and research, and the application of computer technology. Electrical automation means that the machine can be operated automatically, without human control. In fact, industrial production process often can see the combination of electrical automation and artificial intelligence applications. The microscopic point of view, the application of artificial intelligence technology in electrical automation, the system can not only greatly improve the electrical automation equipment, more important is the ability to significantly improve the efficiency of automated production enterprises; From a macro point of view, the combination of electrical automation and artificial intelligence technology, which can not only see the future of intelligent electrical automation development direction, but also reflects the innovation and development of the field of science and technology.
\end{abstract}

\section{An Overview of Artificial Intelligence Technology}

Definition of Artificial Intelligence. The simple definition of artificial intelligence is: By some means, the computer can simulate many kinds of human behavior, and finally make the machine to achieve the same operation effect as the manual operation. The subject looks simple, but in fact incorporates a variety of professional knowledge, including: Mathematics, philosophy, cognitive science, biology, bionics, computer application, information theory, cybernetics, and psychology etc...See from the surface to grasp the discipline of artificial intelligence, the demand is very high, but in fact, the main research direction of artificial intelligence is a comprehensive application of the machine intelligent control, robotics, programming language and image understanding technology etc.. At present, the development of electrical automation from the development level of the requirements of the people still have a certain distance, and the application of artificial intelligence in the field of electric automation will contribute to the development of electrical automation, so close to the requirements of human development in the future, it is of great significance. The perfect combination of artificial intelligence technology and electrical automation will accelerate the innovation and development of electrical automation.

Application Characteristics of Artificial Intelligence. At present, artificial intelligence is the closest to the human brain science and technology, the application of this technology is also more prominent and widely.

This is mainly from the following aspects can be seen:

Information Collection and Processing.

One of the major characteristics of artificial intelligence is to replace the human some complex mental work, while ensuring the accuracy, it can save a lot of work time, and the efficiency is significantly improved. For example, often with the help of some computer programming software, to complete some of our repeated and tedious information collection of data. At the same time, 
these procedures can also be collected to the information data processing, and spend less time to get a similar result with the human brain processing, high efficiency.

System Automatic Monitoring Function.

When the electronic automation system works, the system of artificial intelligence technology can be used to monitor the running state of the working equipment in real time. Not only can check whether the device is working at any time, but also can understand the operation of the device at any time value. When the device is not working properly or running the value beyond the normal range, the system will automatically record such specific events, and automatically reported to the situation. In addition, the system also has the function of monitoring sound and light and image and telephone alarm.

Operation Control Function.

Through the application of artificial intelligence technology, the staff can control the electrical system through the computer program software. For example, the staff through a simple computer operation, you can remotely on the device circuit breaker or isolation switch operation. In addition, the electrical system can also be based on the requirements of the relevant duty personnel, the operator's authority to limit;4. Fault Recording Function. The artificial intelligent system can not only simulate the occurrence of faults, but also can record the change of equipment in real time.

The Use of Artificial Intelligence Technology and the Combination of Electrical Automation Technology Control. In view of the application of artificial intelligence technology, there are many different control methods. The artificial intelligence technology is different, the control method cannot be generalized, it should analyze specific issues, matching the appropriate method for the control of artificial intelligence. Because the processing method of artificial intelligence technology is mainly a function approximation, the intelligent model has the following unique advantages compared with a large number of ordinary function estimator.

Strengthen the Control Performance

Through some of the relevant data of the controller to make some changes, it may be a greater improvement in the operational performance of the equipment machine. For example: the fuzzy logic controller to fine tune the relevant data, not only can make the reaction rate is faster, a certain point of view, the performance of the equipment is to obtain a certain strengthening.

Using the Method is More Simple

In view of the deficiency of the traditional controller, the reasons are analyzed and improved, and the improved artificial intelligence controller is formed. The control difficulty is not only lower, but also has stronger adaptability to new information and data. Simply say, even if the operator is not the corresponding experts, or do not have more professional knowledge, but as long as the understanding of some simple information language, they still have the function of the design.

High Consistency

One of the characteristics of the traditional control method is that it has the specificity. This is both an advantage and a disadvantage. The advantage is that the control operation of a particular object is easier to implement. But for other control objects, this control method may not be applicable, and its control effect will be greatly reduced. However, compared with the traditional control method, artificial intelligence has a high degree of consistency, improve the traditional single control algorithm, in the face of various types of data input, artificial intelligence can ensure uniform height control effect.

\section{Application of Artificial Intelligence Technology in Electrical Automation Control}

In recent years, with the rapid development of the technology of artificial intelligence, more and more attention has been obtained, and the corresponding demand of the market is higher and higher. Scientists have also paid more and more attention to the application and development of this new product. At present, the design of artificial intelligence products constantly refreshes the traditional concepts, and even some of the artificial intelligence products to complete the task of ordinary people cannot do. In industrial production, artificial intelligence technology can be used in electrical automation control. For example, when the electrical automation control system is running, there is 
a failure problem, the artificial intelligence not only can make the corresponding prediction and protection of the product, but also can reasonably optimize the control of product production.

Optimization of Product Design. Traditional electrical products design ideas are often relatively simple, this design is relatively simple, when the product design is usually designed to try to design a variety of products through a variety of experiments. Thus, the traditional design technology content is relatively low, and does not require more skill, the design process is more complicated and trivial, need more time, it is easy to reduce the efficiency of work, and the final design of electrical products is usually not optimal design. Today's social economy has been a certain development, the national development of science and technology is also more and more attention. With the continuous investment of science and technology research and development funds, the level of domestic technology in recent years has been rapid upgrade, as the field of artificial intelligence has also been a breakthrough research results. In the design of electrical products, China has gradually shifted from the traditional way of thinking to the direction of intelligent production. Compared with the traditional manual mode of production, intelligent mode of production is not only faster, but also the production of higher precision. This mode of production not only greatly reduced the production time of enterprises, but also to enhance the quality of production, will be conducive to the rapid development of enterprises.

Monitoring and Handling of Accidents and Failures. Although in the course of the operation of the electrical automation system, equipment failure and the occurrence of artificial accidents usually rarely occur, but it is difficult to avoid. When these two conditions occur, compared with other methods, artificial intelligence technology has a better treatment method in this aspect, especially has a unique advantage for the engine, the disposal of the fault voltage. For example, a transformer operation occurred in the process of failure. According to the traditional processing method is, First, the gas samples were collected, and then the samples were separated and analyzed. Finally, the possible reasons for the failure of the machine were obtained based on the analysis results. Using this approach will lead to excessive maintenance process of human resources and material resources, and will waste a lot of time in the maintenance process. However, the probability of the final correct diagnosis is not high. Even often appear to be misdiagnosed, the misdiagnosis will bring great loss to the enterprise. In the face of these problems of prevention and treatment of accident or failure, compared with the traditional processing method, processing method, the application of artificial intelligence technology, superior. When a fault occurs, the system will automatically guide the related technical experts will be based on the machine fault of longitudinal contrast, after the comparative analysis, find out the possible causes of failure, and timely solutions to the matching problem. From then on, the processing method of artificial intelligence technology is applied to the fault diagnosis accuracy rate is very high, and greatly shorten the time of equipment maintenance, greatly improve the processing efficiency of the fault problem.

Simplify the Control Flow of Electrical Automation. Relative to the general production process, the electrical automation production process to be more complex, and the production requirements are extremely strict. For example, electrical automation in the production process, because of some reasons causing a step in production, there has been little operational errors, which may lead to the entire production process is disturbed, even the whole production stops. From now on, once the production process of such problems, will be likely to cause major accidents, and ultimately to bring significant economic losses to enterprises. So the researchers attach great importance to the normal operation of electrical equipment, and the application of artificial intelligence technology in production will help to solve this problem, the artificial intelligence technology can greatly simplify the electrical automation control process. For traditional methods, artificial intelligence has a unique advantage in this regard. In the daily production process, the artificial intelligence will carry on the real-time collection, the reorganization, the analysis and the record to the equipment working information. When the equipment failure, the system automatically searches for similar fault cases, based on the reference case, the best solution to quickly match. This greatly simplifies the maintenance process of equipment failure, not only 
improve the accuracy of fault maintenance, optimize the control flow of electrical automation. It also saves precious time to repair, as far as possible to avoid delay in production, and greatly reduce the cost of enterprise's maintenance and accident loss.

\section{Conclusion}

In today's rapid development of science and technology, artificial intelligence technology is also in constant attention and development. In today's social life, we often can see the application of artificial intelligence technology. In industrial production, there are more and more enterprises to increase investment in artificial intelligence technology, artificial intelligence technology and electrical automation control combined with the application of very successful, can greatly improve the production quality and production efficiency.

Generally speaking, the application of artificial intelligence technology in electrical automation control has broad prospects for development. If the artificial intelligence technology is fully applied to the industrial production, it will make up for the deficiency of the simple electric automation control, and greatly improve the operation efficiency of the electric automation.

\section{Reference}

[1] Feng Jin. Analysis on the application of artificial intelligence technology in electrical automation control $[\mathrm{J}]$. Electronic technology and software engineering. 2014 (01)

[2] Ma Zhongxiong. On the electrical automatic control of the artificial intelligence technology of [J]. Electronic technology and software engineering, 2014 (11).

[3] Hu Kaibao , moor-jankowski TaoQing. Corpus and translation studies: trends and problems -- 2007 international academic seminar of corpus translation studies, and research review [J]. Journal of foreign languages (journal of Shanghai international studies university), 2007 (05)

[4] nai-xing wei. John Sinclair linguistic heritage - on the thought and method of [J]. Journal of foreign languages (journal of Shanghai international studies university), 2007 (04)

[5] Miss Ann . Small and medium-sized enterprise network construction scheme discussion [J]. Journal of jiangsu science and technology information, 2013, 11:42-43. 